\title{
Valorização de resíduos têxteis na indústria da construção civil
}

\author{
Elen Machado de Oliveira ${ }^{1 *}$ (1) \\ Elis Machado de Oliveira ${ }^{1}$ \\ Camila Machado de Oliveira ${ }^{2}$ \\ Alexandre Gonçalves Dal-Bó ${ }^{1}$ \\ Michael Peterson ${ }^{1}$
}

\section{Resumo}

Argamassas de revestimento foram produzidas com traço de 1:1:6 (cimento:cal hidráulica:areia) com a incorporação de aparas de tecido como material de reforço. As aparas são um resíduo gerado pela indústria têxtil e foram desfiadas manualmente para se assemelharem as fibras existentes no mercado. Para analisar sua influência nas propriedades das argamassas de revestimento, foi realizado um planejamento fatorial $3^{2}$, variando o teor de fios em 1,2 e $3 \%$, em relação à massa de aglomerante, e o comprimento em 1,2 e $3 \mathrm{~cm}$. As argamassas foram testadas nos ensaios de resistência à compressão, massa específica, índice de vazios e absorção de água. O resíduo, quando introduzido nas argamassas, elevou a relação água/aglomerante para o índice de consistência fixado em $260 \pm 5 \mathrm{~mm}$. Em relação à referência, a resistência à compressão das argamassas com os fios do tecido foi inferior, mas atendeu ao mínimo estabelecido em norma. Essa redução está associada à quantidade de água adicionada à mistura. A incorporação do resíduo reduziu a massa específica das argamassas e, em uma situação prática, contribuiria para revestimentos mais leves.

Palavras-chave: Argamassa de revestimento; Resíduo têxtil; Material de reforço; Resistência mecânica.

\section{Textile waste valorization in the construction industry}

\begin{abstract}
Coating mortars with a trace of 1:1:6 (cement:hydraulic lime:sand) were produced with the incorporation of fabric shavings as reinforcement material. The shavings are a waste generated by the textile industry and were shredded manually to resemble the fibers on the market. In order to analyze its influence on the properties of coating mortars, a $3^{2}$ factorial design was carried out varying the yarn content in 1,2 and 3\%, in relation to the binder mass, and the length in 1, 2 and $3 \mathrm{~cm}$. Mortars were evaluated by compressive strength, specific mass, void index and water absorption tests. The waste, when introduced into the mortars, increased the water/binder ratio to the consistency index set at $260 \pm 5 \mathrm{~mm}$. Compared to the reference, the compressive strength of mortars with the fabric yarns was lower, but met the minimum established in the standard. This reduction is associated with the amount of water added to the mixture. The incorporation of the waste reduced the specific mass of mortars and, in a practical situation, would contribute to lighter coatings.
\end{abstract}

Keywords: Coating mortar; Textile waste; Reinforcement material; Mechanical strength.

\section{Introdução}

A indústria têxtil e de confecção surgiu da necessidade humana de vestuário. Presente em todos os países, tem muita influência na economia, incluindo a brasileira, por gerar empregos, ter grande volume de produção e ser crescente o número de exportações [1].
Com grande relevância no cenário mundial, o Brasil tem a quinta maior indústria têxtil do mundo, segundo a Associação Brasileira da Indústria Têxtil e de Confecção [2]. Em 2017, o país possuía 27.500 empresas formais e uma produção média de 1,3 milhão de toneladas e 8,9 bilhões de

${ }^{1}$ Programa de Pós-Graduação em Ciência e Engenharia de Materiais - PPGCEM, Universidade do Extremo Sul Catarinense - UNESC, Criciúma, SC, Brasil.

2 Programa de Pós-Graduação em Ciência e Engenharia de Materiais - PGMAT, Universidade Federal de Santa Catarina - UFSC, Florianópolis, SC, Brasil.

*Autor correspondente: elen0407@hotmail.com 
peças. Com 1,5 milhão de trabalhadores de forma direta e mais de 8 milhões quando contabilizados os indiretos, representa $16,7 \%$ dos empregos e 5,7\% do faturamento, sendo a segunda maior empregadora da indústria de transformação [3].

Santa Catariana tem grande representatividade nacional no setor, com $20,04 \%$ na parte têxtil e $22,27 \%$ no vestuário. A região sul se destaca no segmento de confecções, juntamente com a do Vale do Itajaí e do Norte, contribuindo para que o estado seja o segundo polo têxtil e de vestuário do Brasil [4].

$\mathrm{Na}$ produção de vestuário, são geradas grandes quantidades de resíduos resultantes principalmente da etapa de corte nas confecções. A falta de qualificação de mão de obra afeta diretamente esse desperdício. Esses resíduos são descartados em lixões e aterros sem qualquer tipo de controle, sendo alguns incinerados, processo este altamente poluidor [5].

No Brasil, são geradas 175.000 toneladas por ano de resíduos dessa etapa do processo, segundo o Sindicato das Indústrias de Fiação e Tecelagem do Estado de São Paulo [6], e apenas 36.000 , equivalente a $20 \%$, são reutilizados para produzir mantas, barbantes, novas peças de roupa e fios [7]. Os demais são encaminhados para aterros sanitários ou são descartados de forma incorreta [8].

Para valorizar os resíduos industrias e assim estimular os princípios sociais em relação ao desenvolvimento sustentável, é necessário desenvolver soluções para transformá-los em recursos [9]. A construção civil é um dos setores com maior capacidade para absorver essa grande quantidade de resíduos. A incorporação desses materiais, em algumas situações, pode melhorar determinadas propriedades do produto final [10].

Nas últimas duas décadas, a tecnologia moderna se dedicou ao estudo de métodos para melhorar o desempenho de materiais cimentícios. $\mathrm{O}$ aumento da resistência à compressão de concretos e argamassas eleva a sua fragilidade, tornando crescente o uso de fibras como material de reforço para proporcionar um ganho de resistência à tração e ductilidade [11].

Além disso, a utilização das fibras aumenta significativamente a tenacidade à fratura e o desempenho pós-fissuração da mistura, reduz a largura e a quantidade de fissuras pela ação da ponte de transferência de tensão, melhorando assim a durabilidade da matriz cimentícia [12].
Baseado neste contexto, avaliou-se a incorporação de aparas de tecido da indústria de confecção como material de reforço na produção de argamassa de revestimento.

\section{Materiais e metódos}

As aparas de tecido foram coletadas em uma empresa de confecção localizada no município de Nova Veneza/SC. Para assemelharem-se às fibras existentes no mercado, os resíduos de tecido foram desfiados manualmente, retornando à forma de fios, e cortados em comprimentos de 1,2 e $3 \mathrm{~cm}$.

Os fios do tecido apresentaram resistência à tração de $78,04 \pm 2,14 \mathrm{MPa}$, massa específica de 1,56 $\pm 0,04 \mathrm{~g} / \mathrm{cm}^{3}$, com um diâmetro médio de $0,5 \mathrm{~mm}$, sendo compostos por celulose.

Para a produção das argamassas de revestimento, foram utilizados o cimento CP IV 32, a cal hidráulica HL2 e uma areia padronizada do Instituto de Pesquisas Tecnológicas (IPT), conforme a ABNT NBR 7214 [13].

As caracterizações do cimento, da cal hidráulica e da areia encontram-se na Tabela 1 .

Para a confecção das argamassas, foi adotado o traço 1:1:6 (cimento:cal hidráulica:areia) em massa e adaptou-se a relação água/aglomerante para um índice de consistência fixado em $260 \pm 5 \mathrm{~mm}$, segundo a ABNT NBR 13276 [14]. $\mathrm{O}$ agregado miúdo utilizado foi uma mistura da mesma quantidade das areias normais 16, 30, 50 e 100.

Os teores de fios do tecido incorporados em relação à massa de aglomerante foram de 1,2 e 3\%, com comprimentos de 1,2 e $3 \mathrm{~cm}$.

Para avaliar a influência desses parâmetros sobre cada variável resposta, executou-se o planejamento estatístico fatorial $3^{\mathrm{k}}$ com dois fatores $\left(3^{2}\right)$ da Tabela 2 . As variáveis respostas foram a resistência à compressão, absorção de água, índice de vazios e massa específica.

O processo de mistura para produção das argamassas aconteceu como determina a ABNT NBR 16541 [15]. Secou-se o agregado miúdo em estufa na temperatura de $105 \pm 5^{\circ} \mathrm{C}$ por 24 horas antes do procedimento. Os fios do tecido foram adicionados no final da mistura e após verificou-se o índice de consistência.

Tabela 1. Caracterização do cimento, da cal hidráulica e da areia

\begin{tabular}{lccc}
\hline \multicolumn{1}{c}{ Caracterização } & \multicolumn{2}{c}{ Materiais } \\
\cline { 2 - 4 } & Cimento & Cal hidráulica & Areia \\
\hline Massa específica $\left(\mathrm{g} / \mathrm{cm}^{3}\right)$ & $2,78 \pm 0,01$ & $2,68 \pm 0,01$ & $2,66 \pm 0,01$ \\
Massa unitária $\left(\mathrm{kg} / \mathrm{m}^{3}\right)$ & - & 582,36 & $1.513,76$ \\
$\mathrm{D}_{90}(\mu \mathrm{m})$ & 34,78 & 43,35 & - \\
$\mathrm{D}_{50}(\mu \mathrm{m})$ & 13,30 & 10,31 & - \\
$\mathrm{D}_{10}(\mu \mathrm{m})$ & 2,25 & 1,47 & - \\
Diâmetro médio $(\mu \mathrm{m})$ & 16,30 & 16,86 & - \\
Absorção de água $(\%)$ & - & - & 0,52 \\
Dimensão máxima característica $(\mathrm{mm})$ & - & - & 2,36 \\
Módulo de finura & - & - & 2,42 \\
\hline
\end{tabular}


$\mathrm{O}$ ensaio de resistência à compressão foi realizado como estabelece a ABNT NBR 13279 [16]. Utilizou-se para romper os 10 corpos de prova de cada composição da Tabela 2 e a de referência (sem resíduo) a prensa EMIC PC200CS, aplicando uma carga uniforme e sem choque de $500 \pm 50 \mathrm{~N} / \mathrm{s}$.

O ensaio para avaliar a absorção de água e determinar o coeficiente de capilaridade seguiu a ABNT NBR 15259 [17]. Após 28 dias de cura, 3 corpos de prova de cada composição foram imersos em água no nível de $5 \pm 1 \mathrm{~mm}$ acima da face submersa. Passados 10 minutos, após a retirada do excesso de água, foram pesados e imersos novamente. O processo de pesagem foi repetido aos 90 minutos.

Realizaram-se todas as etapas do procedimento para determinar o índice de vazios e massa específica nos 5 corpos de prova de cada composição de acordo com a ABNT NBR 9778 [18].

Para o tratamento estatístico dos dados, empregou-se o software Statistica 13.0 Trial Version. Nas análises, utilizaram-se os resultados de todos os corpos de prova, não somente a média para cada composição.

\section{Resultados e discussão}

A relação água/aglomerante e o índice de consistência de todas as misturas estudadas encontram-se na Tabela 3.

Tabela 2. Matriz de dados do planejamento fatorial $3^{2}$

\begin{tabular}{ccccc}
\hline \multirow{2}{*}{ Experimento } & Níveis & $\begin{array}{c}\text { Feor de fios } \\
\text { (\%) }\end{array}$ & $\begin{array}{c}\text { Comprimento } \\
\text { (cm) }\end{array}$ \\
\hline $\mathrm{T} 1 \mathrm{C} 1$ & -1 & -1 & 1 & 1 \\
$\mathrm{~T} 1 \mathrm{C} 2$ & -1 & 0 & 1 & 2 \\
$\mathrm{~T} 1 \mathrm{C} 3$ & -1 & 1 & 1 & 3 \\
$\mathrm{~T} 2 \mathrm{C} 1$ & 0 & -1 & 2 & 1 \\
$\mathrm{~T} 2 \mathrm{C} 2$ & 0 & 0 & 2 & 2 \\
$\mathrm{~T} 2 \mathrm{C} 3$ & 0 & 1 & 2 & 3 \\
$\mathrm{~T} 3 \mathrm{C} 1$ & 1 & -1 & 3 & 1 \\
$\mathrm{~T} 3 \mathrm{C} 2$ & 1 & 0 & 3 & 2 \\
$\mathrm{~T} 3 \mathrm{C} 3$ & 1 & 1 & 3 & 3 \\
\hline
\end{tabular}

Analisando os valores da relação água/aglomerante, para o índice de consistência fixado em $260 \pm 5 \mathrm{~mm}$, as misturas com a adição de fios do tecido necessitaram de maior quantidade de água, pois o resíduo a absorve e dificulta o espalhamento das argamassas.

A Figura 1 mostra os resultados de todos os ensaios de cada composição.

A Tabela 4 mostra a análise de variância para o índice de vazios das argamassas.

Embora o fator comprimento (L) e as interações teor de fios $(\mathrm{Q}) \times$ comprimento $(\mathrm{L})$ e teor de fios $(\mathrm{Q}) \times$ comprimento (Q) não sejam significativos para a variável resposta índice de vazios ( $p>\alpha$, para $\alpha=0,05)$, quando não considerados, o ajuste do modelo foi prejudicado (redução do $\mathrm{R}^{2}$ ).

A superfície de resposta da Figura 2a indica que o aumento do índice de vazios das argamassas está relacionado com o acréscimo de fios e que o comprimento destes fios tem menor influência sobre esta propriedade.

Esse aumento do índice de vazios pode estar ligado à relação água/aglomerante das misturas, pois aquelas com o acréscimo do resíduo precisaram de uma maior quantidade de água para manter a trabalhabilidade. Quando a água é consumida no processo de hidratação ou evaporada, deixa espaços vazios [19].

No processo de mistura das argamassas, para os teores de fios de 1 e $2 \%$ foi utilizada a mesma proporção de água/aglomerante para os três comprimentos e, por isto, não se observa grande diferença entre os valores de índice de vazios na superfície de resposta. Já para o teor de fios de 3\%, a mesma quantidade de água foi adicionada para os tamanhos de 1 e $3 \mathrm{~cm}$, mas o volume foi menor para o comprimento de $2 \mathrm{~cm}$, provocando o abaloamento da superfície de resposta da Figura 2a.

Mínguez, Vicente e González [19] verificaram o aumento do índice de vazios com a presença de fibras de aço, mesmo com proporções iguais de água nas argamassas de referência e nas com fibra de aço, pois o material de reforço retém mais o ar.

Rosato et al. [20] trabalharam com argamassa com proporção cal hidráulica natural NHL3,5:areia de 1:2 e fibras de celulose nos teores de $0,1,0,25$ e $0,5 \%$ em relação

Tabela 3. Índice de consistência das misturas

\begin{tabular}{ccccc}
\hline Experimento & Teor de fios (\%) & Comprimento (cm) & $\begin{array}{c}\text { Relação água/ } \\
\text { aglomerante }\end{array}$ & $\begin{array}{c}\text { Índice de consistência } \\
( \pm \mathbf{0 , 0 1} \mathbf{~ m m})\end{array}$ \\
\hline Referência & - & - & 0,70 & 261,03 \\
T1C1 & 1 & 1 & 0,80 & 258,42 \\
T1C2 & 1 & 2 & 0,80 & 256,03 \\
T1C3 & 1 & 3 & 0,80 & 255,78 \\
T2C1 & 2 & 1 & 0,85 & 256,27 \\
T2C2 & 2 & 2 & 0,85 & 260,65 \\
T2C3 & 2 & 3 & 0,85 & 255,78 \\
T3C1 & 3 & 1 & 0,92 & 255,28 \\
T3C2 & 3 & 2 & 0,90 & 256,15 \\
T3C3 & 3 & 3 & 0,92 & 256,12 \\
\hline
\end{tabular}




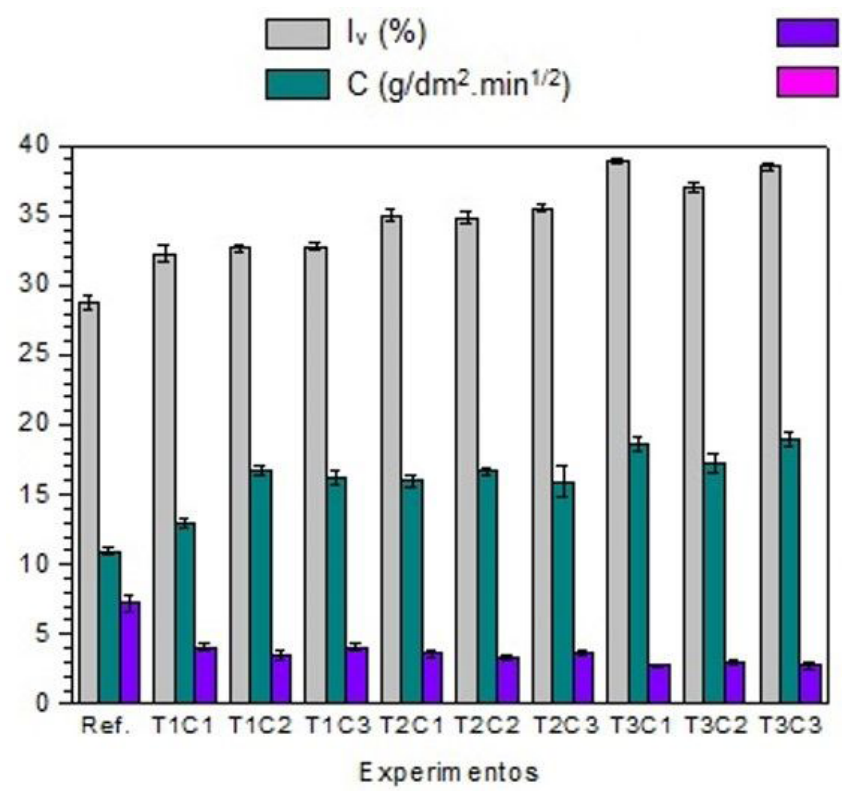

$$
\begin{aligned}
& R_{0}(\mathrm{MPa}) \\
& \rho_{s}\left(\mathrm{~g} / \mathrm{cm}^{3}\right)
\end{aligned}
$$

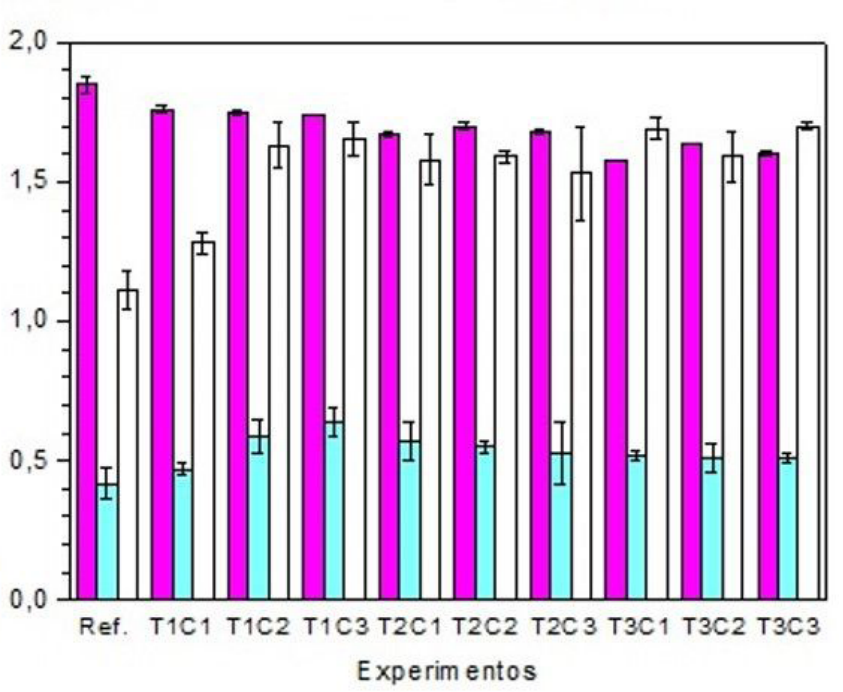

Figura 1. Índice de vazios $\left(\mathrm{I}_{\mathrm{y}}\right)$, massa específica $\left(\mathrm{\rho}_{\mathrm{s}}\right)$, absorção de água por capilaridade $\left(\mathrm{A}_{10}\right.$ e $\left.\mathrm{A}_{90}\right)$, coeficiente de capilaridade $(\mathrm{C})$ e resistência à compressão $\left(\mathrm{R}_{\mathrm{c}}\right)$ dos experimentos.

(a)

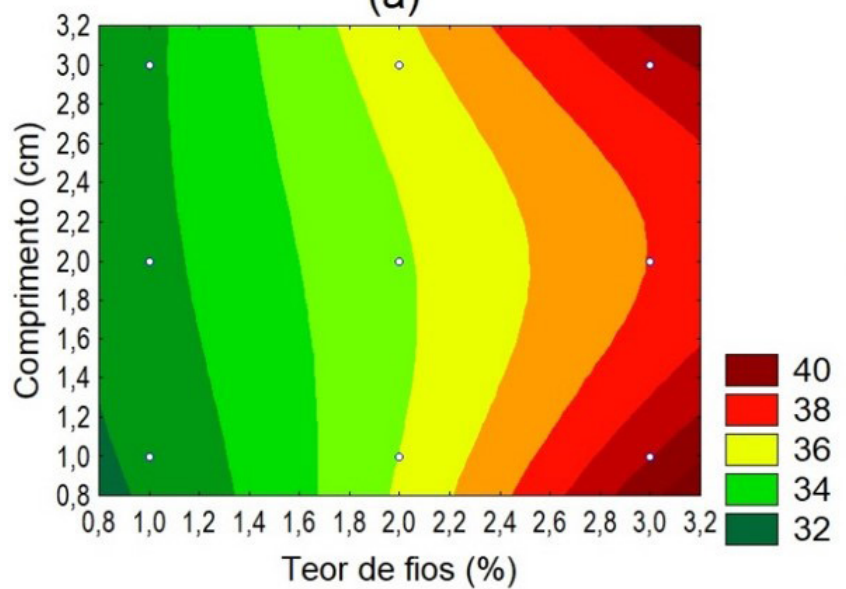

(c)

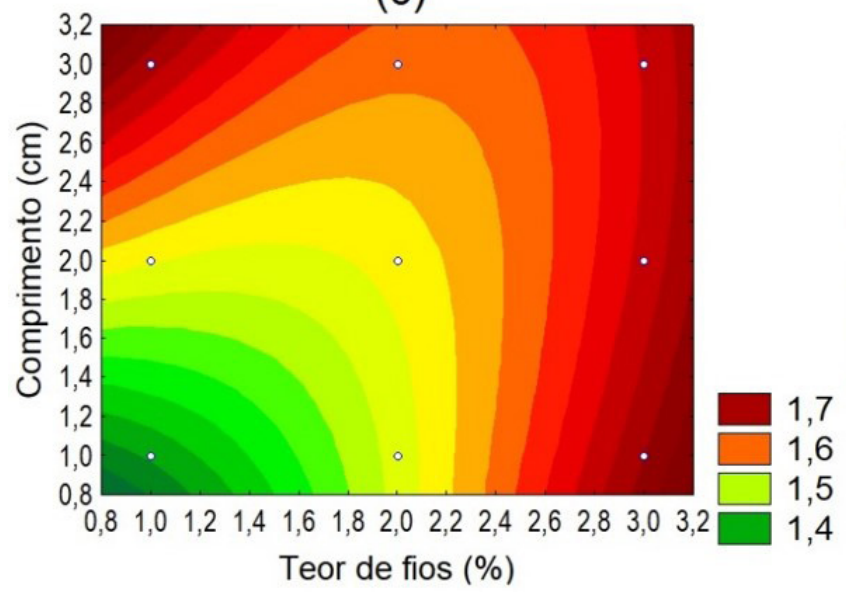

(b)

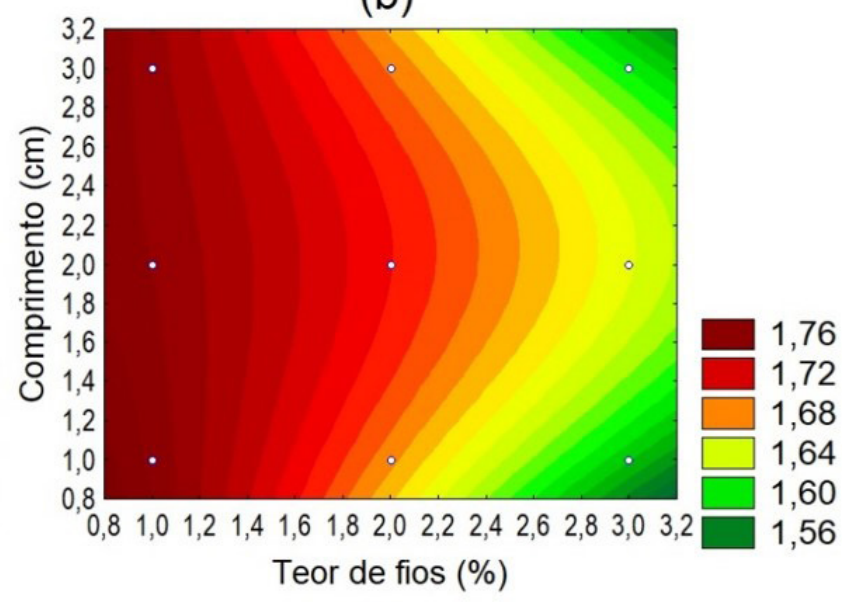

(d)

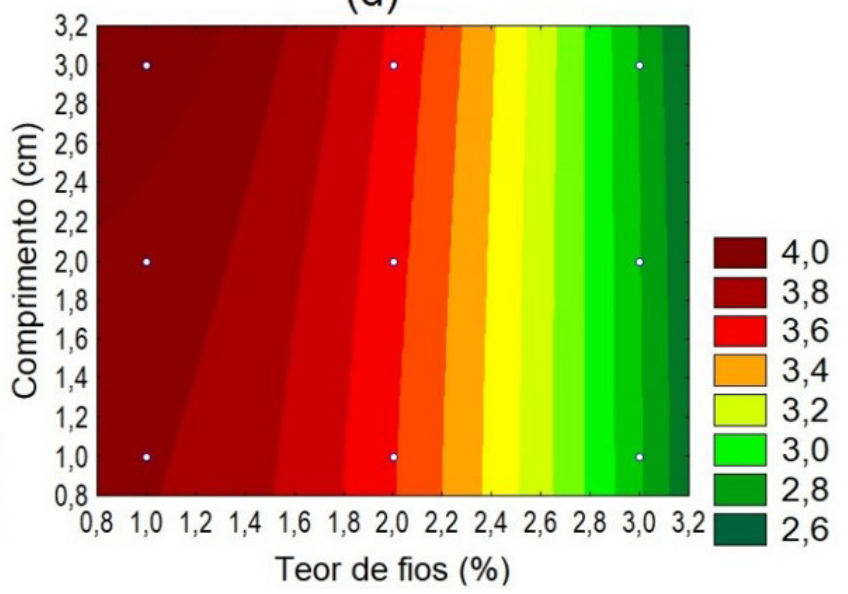

Figura 2. Superfície de resposta para o (a) índice de vazios, (b) massa específica, (c) absorção de água por capilaridade aos 90 minutos e (d) resistência à compressão. 
à quantidade de massa de água, sendo que na mistura de referência foram utilizados 342 gramas de água e nas outras 352, 362 e 372 gramas, respectivamente, mantendo a trabalhabilidade entre 160 e $170 \mathrm{~mm}$. Os autores observaram um incremento na porosidade das argamassas de $26,38 \%$ com $0,1 \%$ de fibra de celulose para $29,09 \%$ com $0,5 \%$.

A Tabela 5 traz a análise de variância para a massa específica das argamassas. Entre os fatores que não foram significativos para a variável resposta, excluiu-se apenas aquele que, quando desconsiderado, não prejudicou o ajuste do modelo - a interação entre o teor de fios $(\mathrm{Q})$ e o comprimento (L).

Como o índice de vazios e a massa específica são inversamente proporcionais, avaliando a superfície de resposta da Figura 2b, os menores valores de massa específica são observados para as argamassas com maiores teores de fios e maiores índices de vazios (segundo a Figura 2a). Essa relação também fica evidente na semelhança entre os formatos das superfícies de respostas para as duas variáveis.

Além disso, como a massa específica dos fios $\left(1,56 \pm 0,04 \mathrm{~g} / \mathrm{cm}^{3}\right)$ é menor que a do cimento $\left(2,78 \pm 0,01 \mathrm{~g} / \mathrm{cm}^{3}\right)$, da cal hidráulica $\left(2,68 \pm 0,01 \mathrm{~g} / \mathrm{cm}^{3}\right)$ e da areia $\left(2,66 \mathrm{~g} / \mathrm{cm}^{3}\right)$, ela contribui para a redução da massa específica das argamassas com maiores quantidades de resíduo.

Coppola et al. [21] destacam a importância do uso de materiais mais leves nas construções por possibilitarem a redução das cargas na estrutura.

Todas as argamassas foram classificadas pelo coeficiente de capilaridade na maior categoria da norma ABNT NBR 13281 [22] - maior que $10,0 \mathrm{~g} / \mathrm{dm}^{2} \cdot \mathrm{min}^{1 / 2}$.

A Tabela 6 demonstra a análise de variância para a absorção de água por capilaridade aos 90 minutos.

Os melhores valores de $\mathrm{R}^{2}(0,73283)$ e $\mathrm{R}^{2}$ ajustado $(0,66922)$ foram alcançados ao se desconsiderar os fatores teor de fios $(\mathrm{Q})$ e comprimento $(\mathrm{Q})$ e a interação entre o teor de fios $(\mathrm{Q})$ e o comprimento $(\mathrm{Q})$, que não foram significativos para a variável resposta.

Analisando a superfície de resposta da Figura 2c, a absorção de água por capilaridade é menor para as amostras com o menor teor de fios do tecido e menor comprimento, sendo que para 1 e $2 \%$ de adição, o tamanho dos fios interferiu de forma negativa. Como a absorção de água se deu por capilaridade, ela foi facilitada por comprimentos de fios maiores.

Tabela 4. Análise de variância (ANOVA) para o índice de vazios. Onde 1 - teor de fios, 2 - comprimento, L - variável linear e Q - variável quadrática

\begin{tabular}{cccccc}
\hline Fator & SS & df & MS & F & p \\
\hline Teor de fios (L) & $\mathbf{2 3 4 , 0 2 5 5}$ & $\mathbf{1}$ & $\mathbf{2 3 4 , 0 2 5 5}$ & $\mathbf{1 9 2 4 , 4 1 1}$ & $\mathbf{0 , 0 0 0 0 0 0}$ \\
Teor de fios (Q) & $\mathbf{0 , 5 0 3 3}$ & $\mathbf{1}$ & $\mathbf{0 , 5 0 3 3}$ & $\mathbf{4 , 1 3 8}$ & $\mathbf{0 , 0 4 9 3 4 1}$ \\
Comprimento (L) & 0,3499 & 1 & 0,3499 & 2,877 & 0,098462 \\
Comprimento (Q) & $\mathbf{4 , 3 4 7 2}$ & $\mathbf{1}$ & $\mathbf{4 , 3 4 7 2}$ & $\mathbf{3 5 , 7 4 7}$ & $\mathbf{0 , 0 0 0 0 0 1}$ \\
$\mathbf{1 L} \times \mathbf{2 L}$ & $\mathbf{1 , 1 3 2 9}$ & $\mathbf{1}$ & $\mathbf{1 , 1 3 2 9}$ & $\mathbf{9 , 3 1 6}$ & $\mathbf{0 , 0 0 4 2 5 2}$ \\
$\mathbf{1 L} \times \mathbf{2 Q}$ & $\mathbf{5 , 8 0 3 3}$ & $\mathbf{1}$ & $\mathbf{5 , 8 0 3 3}$ & $\mathbf{4 7 , 7 2 1}$ & $\mathbf{0 , 0 0 0 0 0 0}$ \\
1Q $\times 2 \mathrm{~L}$ & 0,3840 & 1 & 0,3840 & 3,158 & 0,084022 \\
$1 \mathrm{Q} \times 2 \mathrm{Q}$ & 0,2961 & 36 & 0,2961 & 2,434 & 0,127442 \\
Erro & 4,3779 & 44 & 0,1216 & & \\
Total SS & 251,2200 & & & & \\
$\mathrm{R}^{2}$ & 0,98257 & & & & \\
$\mathrm{R}^{2}$ ajustado & 0,9787 & & &
\end{tabular}

Tabela 5. Análise de variância para a massa específica

\begin{tabular}{cccccc}
\hline Fator & SS & df & MS & F & p \\
\hline Teor de fios (L) & $\mathbf{0 , 1 5 4 0 8 3}$ & $\mathbf{1}$ & $\mathbf{0 , 1 5 4 0 8 3}$ & $\mathbf{3 6 5 0 , 6 4 0}$ & $\mathbf{0 , 0 0 0 0 0 0}$ \\
Teor de fios (Q) & $\mathbf{0 , 0 0 0 2 5 0}$ & $\mathbf{1}$ & $\mathbf{0 , 0 0 0 2 5 0}$ & $\mathbf{5 , 9 2 3}$ & $\mathbf{0 , 0 1 9 8 9 1}$ \\
Comprimento (L) & 0,000083 & 1 & 0,000083 & 1,974 & 0,168324 \\
Comprimento (Q) & $\mathbf{0 , 0 0 6 2 5 0}$ & $\mathbf{1}$ & $\mathbf{0 , 0 0 6 2 5 0}$ & $\mathbf{1 4 8 , 0 7 9}$ & $\mathbf{0 , 0 0 0 0 0 0}$ \\
$\mathbf{1 L} \times \mathbf{2 L}$ & $\mathbf{0 , 0 0 1 1 2 5}$ & $\mathbf{1}$ & $\mathbf{0 , 0 0 1 1 2 5}$ & $\mathbf{2 6 , 6 5 4}$ & $\mathbf{0 , 0 0 0 0 0 9}$ \\
$\mathbf{1 L} \times \mathbf{2 Q}$ & $\mathbf{0 , 0 0 4 6 8 2}$ & $\mathbf{1}$ & $\mathbf{0 , 0 0 4 6 8 2}$ & $\mathbf{1 1 0 , 9 2 1}$ & $\mathbf{0 , 0 0 0 0 0 0}$ \\
$\mathbf{1 Q} \times \mathbf{2 Q}$ & $\mathbf{0 , 0 0 0 2 4 5}$ & $\mathbf{1}$ & $\mathbf{0 , 0 0 0 2 4 5}$ & $\mathbf{5 , 8 0 5}$ & $\mathbf{0 , 0 2 1 0 7 6}$ \\
Erro & 0,001562 & 37 & 0,000042 & & \\
Total SS & 0,168280 & 44 & & & \\
$\mathrm{R}^{2}$ & 0,99072 & & & & \\
$\mathrm{R}^{2}$ ajustado & 0,98896 & & & & \\
$\alpha$ & 0,05 & & & & \\
\hline
\end{tabular}


Tabela 6. Análise de variância para a absorção de água por capilaridade aos 90 minutos

\begin{tabular}{cccccc}
\hline Fator & SS & df & MS & F & p \\
\hline Teor de fios (L) & $\mathbf{0 , 0 8 6 8 0 6}$ & $\mathbf{1}$ & $\mathbf{0 , 0 8 6 8 0 6}$ & $\mathbf{1 3 , 4 5 7 4 9}$ & $\mathbf{0 , 0 0 1 4 3 1}$ \\
Comprimento (L) & $\mathbf{0 , 0 5 5 5 5 6}$ & $\mathbf{1}$ & $\mathbf{0 , 0 5 5 5 5 6}$ & $\mathbf{8 , 6 1 2 7 9}$ & $\mathbf{0 , 0 0 7 9 1 5}$ \\
$\mathbf{1 L} \times \mathbf{2 L}$ & $\mathbf{0 , 0 9 3 6 3 3}$ & $\mathbf{1}$ & $\mathbf{0 , 0 9 3 6 3 3}$ & $\mathbf{1 4 , 5 1 6 0 0}$ & $\mathbf{0 , 0 0 1 0 2 2}$ \\
$\mathbf{1 L} \times \mathbf{2 Q}$ & $\mathbf{0 , 0 7 4 7 1 1}$ & $\mathbf{1}$ & $\mathbf{0 , 0 7 4 7 1 1}$ & $\mathbf{1 1 , 5 8 2 4 8}$ & $\mathbf{0 , 0 0 2 6 7 7}$ \\
$\mathbf{1 Q} \times \mathbf{2 L}$ & $\mathbf{0 , 0 6 0 8 4 4}$ & $\mathbf{1}$ & $\mathbf{0 , 0 6 0 8 4 4}$ & $\mathbf{9 , 4 3 2 7 3}$ & $\mathbf{0 , 0 0 5 7 9 4}$ \\
Erro & 0,135457 & 21 & 0,006450 & & \\
Total SS & 0,507007 & 26 & & & \\
$\mathrm{R}^{2}$ & 0,73283 & & & & \\
$\mathrm{R}^{2}$ ajustado & 0,66922 & & & & \\
$\alpha$ & 0,05 & & &
\end{tabular}

Tabela 7. Análise de variância para a resistência à compressão

\begin{tabular}{cccccc}
\hline Fator & SS & df & MS & F & p \\
\hline Teor de fios (L) & $\mathbf{1 7 , 3 0 2 1 4}$ & $\mathbf{1}$ & $\mathbf{1 7 , 3 0 2 1 4}$ & $\mathbf{2 8 3 , 4 5 1 3}$ & $\mathbf{0 , 0 0 0 0 0 0}$ \\
Teor de fios (Q) & $\mathbf{0 , 7 7 6 1 8}$ & $\mathbf{1}$ & $\mathbf{0 , 7 7 6 1 8}$ & $\mathbf{1 2 , 7 1 5 7}$ & $\mathbf{0 , 0 0 0 5 9 8}$ \\
Comprimento (Q) & $\mathbf{1 , 0 1 2 5 0}$ & $\mathbf{1}$ & $\mathbf{1 , 0 1 2 5 0}$ & $\mathbf{1 6 , 5 8 7 2}$ & $\mathbf{0 , 0 0 0 1 0 4}$ \\
$\mathbf{1 L} \times \mathbf{2 Q}$ & $\mathbf{2 , 1 1 4 7 1}$ & $\mathbf{1}$ & $\mathbf{2 , 1 1 4 7 1}$ & $\mathbf{3 4 , 6 4 4 1}$ & $\mathbf{0 , 0 0 0 0 0 0}$ \\
Erro & 5,18848 & 85 & 0,06104 & & \\
Total SS & 26,39401 & 29 & & & \\
$\mathrm{R}^{2}$ & 0,80342 & & & & \\
$\mathrm{R}^{2}$ ajustado & 0,79417 & & & & \\
$\alpha$ & 0,05 & & & & \\
\hline
\end{tabular}

Já para o teor de 3\%, a influência do comprimento na absorção foi menor. Como o índice de vazios das amostras com essa quantidade de resíduo foi superior ao das demais misturas, ele é que foi o fator determinante nos resultados de absorção por capilaridade. Logo, como o modelo leva em consideração apenas o teor de fios e o comprimento, o $\mathrm{R}^{2}$ mostra que ele explica somente $73,283 \%$ da variável resposta absorção de água aos 90 minutos.

De acordo com os requisitos da ABNT NBR 13281 [22], todas as argamassas atingiram a resistência mínima de 1,5 MPa para a compressão.

A Tabela 7 mostra a análise de variância para a resistência à compressão.

Melhores valores de $\mathrm{R}^{2}(0,80342)$ e $\mathrm{R}^{2}$ ajustado $(0,79417)$ foram atingidos com a exclusão de todas as interações e fator que não foram significativos para a variável resposta.

A superfície de resposta da Figura 2 d mostra que a resistência à compressão das argamassas diminuiu com o acréscimo dos fios e que a propriedade foi pouco afetada pelo comprimento deles.

Como as argamassas com a presença dos fios são mais porosas, por conta da evaporação da água (elevada relação água/aglomerante), seus valores de resistência à compressão diminuíram [23]. Por isso, de acordo com o $\mathrm{R}^{2}$, o modelo explica somente $80,342 \%$ da variável resposta resistência à compressão, já que leva em consideração apenas o teor de fios e o comprimento.

Essa redução também foi observada por Rosato et al. [20], em que os valores foram de 3,27, 2,82 e 2,44 MPa para as argamassas com $0,1,0,25$ e $0,5 \%$ de fibras de celulose, respectivamente.

Outros trabalhos que mantiveram a mesma quantidade de água em todas as misturas, variando os teores de fibras, também verificaram a diminuição da resistência à compressão com o aumento da quantidade de material de reforço $[24,25]$.

\section{Conclusões}

As argamassas de revestimento precisam atingir uma trabalhabilidade adequada para aplicação, para isto, a quantidade de água foi adequada para um índice de consistência fixado em $260 \pm 5 \mathrm{~mm}$. À medida que os fios do tecido foram incorporados à mistura, a relação água/ aglomerante aumentou, pois o resíduo absorve grande parte da água, dificultando o espalhamento da argamassa.

A resistência à compressão das amostras com os fios se mostrou inferior à da referência, estando diretamente relacionada com a quantidade de água adicionada à mistura. Mesmo com essa redução, atingiu-se o valor mínimo estabelecido pela norma (1,5 MPa).

Os resultados demonstraram que os vazios oriundos da evaporação da água e do seu consumo no processo de hidratação, fatores relacionados à exigência da modificação na relação água/aglomerante das misturas, elevaram a absorção de água e o coeficiente de capilaridade das argamassas com a presença do resíduo. 
Além disso, os fios do tecido provocaram um aumento no índice de vazios e consequentemente a redução da massa específica das argamassas. Uma das vantagens da utilização de materiais mais leves como revestimentos nas edificações é a diminuição das cargas nas estruturas, interferindo diretamente no seu custo.

\section{Agradecimentos}

Os autores agradecem à Coordenação de Aperfeiçoamento de Pessoal de Nível Superior (CAPES) e a Fundação de Amparo à Pesquisa e Inovação do Estado de Santa Catarina (FAPESC) pelas bolsas concedidas.

\section{Referências}

1. Fujita RML, Jorente MJ. A Indústria Têxtil no Brasil: uma perspectiva histórica e cultural. ModaPalavra e-Periódico. 2015;8(15):153-174.

2 Associação Brasileira da Indústria Têxtil e de Confecção. Muito mais força para o setor e para o Brasil. São Paulo: ABIT; 2017 [acesso em 12 ago. 2020]. Disponível em: http://www.abit.org.br/cont/quemsomos.

3 Associação Brasileira da Indústria Têxtil e de Confecção. Dados gerais do setor referentes a 2017. São Paulo: ABIT; 2018 [acesso em 12 ago. 2020]. Disponível em: http://www.abit.org.br/cont/perfil-do-setor.

4 Federação das Indústrias do Estado de Santa Catarina. Indústria têxtil e do vestuário de SC. Florianópolis: FIESC; 2014 [acesso em 12 ago. 2020]. Disponível em: http://www2.fiescnet.com.br/web/pt/site_topo/pei/info/textil-e-vestuario.

5 Menegucci F, Merteli L, Camargo M, Vito M. Resíduos têxteis : análise sobre descarte e reaproveitamento nas indústrias de confecção. In: Universidade Federal Fluminense. Congresso Nacional de Excelência em Gestão; 2015 Agosto 13-14; Rio de Janeiro, Brasil. Rio de Janeiro: UFF.

6 Sindicato das Indústrias de Fiação e Tecelagem do Estado de São Paulo. Inclusão social e preservação ambiental por meio da reciclagem de resíduos têxteis. São Paulo: Sinditêxtil [acesso em 12 ago. 2020]. Disponível em: http:// sinditextilsp.org.br/retalho_fashion/site/apresentacao.pdf.

7 Ferreira MD, Costa TN, Teixeira FG, Jacques JJ, Cattani A. Redução de resíduos têxteis por meio de projeto de produto de moda. Design \& Tecnologia. 2015;10:38-44.

8 Zonatti WF, Amaral MC, Gasi F, Baruque-Ramos J, Duleba W. Reciclagem de resíduos do setor têxtil e confeccionista no Brasil: panorama e ações relacionadas. Sustentabilidade em Debate. 2015;6(3):50-69.

9 Junco C, Rodríguez A, Calderón V, Muñoz-Rupérez C, Gutiérrez-González S. Fatigue durability test of mortars incorporating polyurethane foam wastes. Construction \& Building Materials. 2018;190:373-381.

10 Cintra CLD, Paiva AEM, Baldo JB. Argamassas de revestimento para alvenaria contendo vermiculita expandida e agregados de borracha reciclada de pneus - Propriedades relevantes. Cerâmica. 2014;60:69-76.

11 Vantadori S, Carpinteri A, Zanichelli A. Lightweight construction materials: mortar reinforced with date-palm mesh fibres. Theoretical and Applied Fracture Mechanics. 2019;100:39-45.

12 Ralegaonkar R, Gavali H, Aswath P, Abolmaali S. Application of chopped basalt fibers in reinforced mortar: a review. Construction \& Building Materials. 2018;164:589-602.

13. Associação Brasileira de Normas Técnicas. ABNT NBR 7214: Areia normal para ensaio de cimento - especificação. Rio de Janeiro: ABNT; 2015.

14. Associação Brasileira de Normas Técnicas. ABNT NBR 13276: Argamassa para assentamento e revestimento de paredes e tetos - determinação do índice de consistência. Rio de Janeiro: ABNT; 2016.

15. Associação Brasileira de Normas Técnicas. ABNT NBR 16541: Argamassa para assentamento e revestimento de paredes e tetos - preparo da mistura para a realização de ensaios. Rio de Janeiro: ABNT; 2016.

16. Associação Brasileira de Normas Técnicas. ABNT NBR 13279: Argamassa para assentamento e revestimento de paredes e tetos - determinação da resistência à tração na flexão e à compressão. Rio de Janeiro: ABNT; 2005.

17. Associação Brasileira de Normas Técnicas. ABNT NBR 15259: Argamassa para assentamento e revestimento de paredes e tetos - determinação da absorção de água por capilaridade e do coeficiente de capilaridade. Rio de Janeiro: ABNT; 2005.

18. Associação Brasileira de Normas Técnicas. ABNT NBR 9778: Argamassa e concreto endurecidos - determinação da absorção de água, índice de vazios e massa específica. Rio de Janeiro: ABNT; 2009.

19 Mínguez J, Vicente MA, González DC. Pore morphology variation under ambient curing of plain and fiberreinforced high performance mortar at an early age. Construction \& Building Materials. 2019;198:718-731. 
20 Rosato L, Stefanidou M, Milazzo G, Fernandez F, Livreri P, Muratore N, et al. Study and evaluation of nanostructured cellulose fibers as additive for restoration of historical mortars and plasters. Materials Today:

Proceedings. 2017;4:6954-6965.

21 Coppola B, Courard L, Michel F, Incarnato L, Scarfato P, Maio L. Hygro-thermal and durability properties of a lightweight mortar made with foamed plastic waste aggregates. Construction \& Building Materials. 2018;170:200206.

22. Associação Brasileira de Normas Técnicas. ABNT NBR 13281: Argamassa para assentamento e revestimento de paredes e tetos - requisitos. Rio de Janeiro: ABNT; 2005.

23 Kesikidou F, Stefanidou M. Natural fiber-reinforced mortars. Journal of Building Engineering. 2019;25:100786.

24 Ramiréz CP, Merino M R, Arrebola CV, Barriguete AV, Kosior-Kazberuk M. Analysis of the mechanical behaviour of the cement mortars with additives of mineral wool fibres from recycling of CDW. Construction \& Building Materials. 2019;210:56-62.

25 Donnini J, Bellezze T, Corinaldesi V. Mechanical, electrical and self-sensing properties of cementitious mortars containing short carbon fibers. Journal of Building Engineering. 2018;20:8-14.

Recebido em: 26 Nov. 2020

Aceito em: 4 Fev. 2021 